\title{
Implications of the COVID-19 Pandemic on School-Based Agricultural Education Teachers in South Carolina
}

\author{
C. Eck ${ }^{1}$
}

\begin{abstract}
Understanding a complete school-based agricultural education (SBAE) program and the many tasks at hand for the teacher are critical in determining the impacts of the COVID-19 pandemic on SBAE teachers. The human capital theory was used to undergird this study, focusing on the components impacting the effectiveness of SBAE teachers. The purpose of the study was to determine the implications of the COVID-19 pandemic on SBAE teachers in South Carolina (SC). This nonexperimental survey research study reached $46.5 \%$ of SBAE teachers in SC through the electronic distribution of a 27-item survey instrument implementing a then-now design. SBAE teachers in SC felt less prepared to deliver relevant classroom instruction, supervise supervised agricultural experiences - projects, advise FFA members, and train Career and Leadership Development Events teams now than they did prior to the COVID-19 pandemic, while they feel more prepared to teach student virtually than ever before. The pandemic impacted the preparedness and self-efficacy of SBAE teachers, impacting both their career and personal life satisfaction. Moving forward SBAE teachers should evaluate their roles and responsibilities associated with their career and determine how to best bring balance into their lives. Additional research on the roles and responsibilities of SBAE teachers post-pandemic should also be considered.
\end{abstract}

\section{Keywords}

human capital, then-now design, teacher preparedness 


\section{Introduction and Problem Statement}

The World Health Organization declared the COVID-19 outbreak as a global pandemic on March 11, 2020 (World Health Organization, 2020). This announcement cased a ripple effect as schools, businesses and government agents across the country had to adapt normal day-to-day operations to limit in-person contact (Centers for Disease Control and Prevention [CDC], 2020). In the case of schools, K-12 teachers, regardless of subject area, had to modify their instructional strategy overnight to begin teaching using virtual platforms (Daniel, 2020). Unfortunately, most school districts were not prepared for this abrupt shift, leaving students and teachers unprepared for this new instructional delivery method (Daniel, 2020). In contrast to in-person course delivery, many "teachers were devoting what would have been instructional time to tackling technology challenges, whether struggling to get up to speed learning the intricacies of a learning management system or fixing access problems on Zoom calls" (Bushweller, 2020, para. 19).

School-based agricultural education (SBAE) teachers were not immune to these changes, as they also experienced an immediate interruption in their daily functions (Lindner et al., 2020). Although SBAE teachers have a primary teaching responsibility (i.e., classroom/laboratory instruction), they are also tasked with roles associated with their National FFA Organization (FFA) chapter, and students supervised agricultural experiences (SAE) (National FFA, 2015). To effectively deliver a complete program, SBAE teachers assume many roles to engage students in all three components (Terry \& Briers, 2010). These diverse roles require additional time commitments over a traditional classroom teacher (Torres et al., 2008). Understanding a complete SBAE program and the many tasks at hand for the teacher are critical in determining the impacts of the COVID-19 pandemic on SBAE teachers. Given the sudden change in instructional delivery and demand for technological proficiency from SBAE teachers (Lindner et al., 2020), the questions of what implications did the COVID-19 pandemic have on SBAE teachers in South Carolina (SC) to deliver a complete program arose.

\section{Theoretical and Conceptual Framework}

Human capital theory was used to undergird this study, as human capital aims to evaluate the education, skills, experiences, and trainings (Becker, 1964; Little, 2003; Schultz, 1971; Smith, 2010; Smylie, 1996) necessary for gainful employment (Becker, 1964) as an SBAE teacher. An individual's human capital and human capital needs differ based on personal and professional characteristics (Lepak \& Snell, 1999). Wilson and Ball (1996) identify one grand challenge in teacher education as understanding where teachers are and where they want to be, as prospective teachers often come into a teacher education program with years of experience. Additionally, "reform efforts have focused mostly on improving teacher effectiveness and student achievement, ignoring the roles of school organization and teacher job satisfaction, which are critical for student success" (Banerjee et al., 2017, p. 234). Furthermore, Banerjee et al. (2017) conclude the need for career satisfaction and a positive environment as being pivotal factors in overall teaching effectiveness, ultimately leading to student achievement. 
When considering human capital development in agricultural education, Eck et al. (2020) developed the conceptual model of effective teaching for SBAE teachers, which takes into account an individual's current human capital along with the identified components of effective SBAE teachers (i.e., intracurricular engagement, personal dispositions, appreciation for diversity and inclusion, pedagogical preparedness, work-life balance, and professionalism) and personal, professional, and environmental factors (i.e., career tenure, program size, certification pathway, and personal attributes), all of which lead to effective teaching in SBAE. The conceptual model developed by Eck et al. (2020) was adapted for this study to include additional factors related to the ongoing COVID-19 pandemic (i.e., marital status, family, the COVID-19 pandemic, and grade levels taught). Together, the human capital theory and the conceptual model of effective teaching for SBAE teachers (Eck et al., 2020) provides a lens to evaluate the preparedness, self-efficacy, work-life balance, and motivation of SBAE teachers in SC during the COVID-19 pandemic. The factors being evaluated in this study are woven into the human capital (i.e., education, skills, experiences, and trainings) SBAE teachers have developed to be effective in their chosen profession, although the pandemic has caused many teachers to question their preparedness (Bushweller, 2020; Daniel, 2020).

\section{Purpose}

The purpose of the study was to determine the implications of the COVID-19 pandemic on SBAE teachers in SC. Understanding SBAE teachers' perceptions prior to the pandemic and now, amidst the pandemic, will help to inform agricultural teacher educators and other stakeholders of the specific needs in preparing and supporting SBAE teachers. Four objectives directed this study:

1. determine the preparedness of SBAE teachers to deliver a complete SBAE program before and during the COVID-19 pandemic,

2. establish perceived levels of self-efficacy prior to and during the pandemic,

3. determine the work-life balance and satisfaction of SBAE teachers prior to and during the pandemic, and

4. identify the motivational factors driving SBAE teachers in their careers.

\section{Methods}

This non-experimental survey research study aimed to reach SBAE teachers across SC $(N=155)$. The survey frame used publicly available email address for the SBAE teachers, where they received an email invitation to participate in the research study. To increase response rate the tailored design method was followed for electronic survey design and electronic distribution (Dillman et al., 2014), resulting in a $46.5 \%(n=72)$ response rate. With over half of the population of interest not responding (53.5\%), non-response bias was of concern. To address this concern the recommendations of Lindner et al. (2001) where followed and a comparison of early to late respondents was conducted, resulting in no statistically significant differences between the two groups (i.e., early and late respondents). Therefore, the $46.5 \%$ of SBAE 
teachers in SC who responded were considered to be representative on the population of interest. A 27-item researcher developed survey was used for data collection. The instrument was developed using a then-now design to allow participants to reflect on their experiences prior to the COVID-19 pandemic (then), while also reporting their current experiences teaching SBAE during the pandemic (now) on the same items. A then-now design (John \& Robbins, 1994) was chosen due to the nature of the pandemic, as researchers did not anticipate a pandemic to collect data prior to the onset. Prior to distribution, the survey instrument was evaluated for face and content validity by three faculty members in agricultural education at [University], following the recommendations of Privitera (2020). The 27-item survey instrument was divided into eight sections including, preparedness to teach, teaching self-efficacy, hours worked, motivation to teach, work-life satisfaction, instructional delivery, COVID-19 impacts, and personal and professional characteristics.

The preparedness to teach section asked participants to score five-items on a four-point Likerttype scale ( 1 = strongly disagree; 4 = strongly agree) based on their preparedness pre-COVID and currently (during pandemic). Teaching self-efficacy was measured using two-items, asking teachers to indicate their pre-COVID self-efficacy for classroom/laboratory instruction, SAE supervision, and FFA advisement, each on a zero to 100 sliding scale, the second question was the same except teachers reported current (during pandemic) self-efficacy. The third section addressed hours worked pre-COVID and during COVID using two questions, where teachers indicated the average number of hours per week spent lesson planning, teaching, grading, supervising SAE projects, advising the FFA chapter, and on personal/family time. Motivation to teach SBAE was evaluated by asking teachers to rank four-items (i.e., teaching agriculture, advising FFA members, working with SAE projects, and preparing Career and Leadership Development Events [CDE/LDE] teams) based on their motivation, where one is the greatest motivator and four is the least motivating. To address work-life satisfaction, teachers selected their career and life satisfaction both pre-COVID and currently on a five-point Likert-type scale with 1 being extremely dissatisfied and 5 being extremely satisfied. Three questions addressed instructional delivery, including "how are you currently delivering classroom instruction?", "did you ever deliver online instruction prior to the COVID-19 pandemic?" and "do you feel prepared to deliver online instruction?". To determine COVID-19 impacts on SBAE, teachers indicated their greatest challenges, greatest benefits, and how [University] could further support them moving forward. The final section of the survey instrument included nine personal and professional demographic questions (i.e., sex, ethnicity, age, marital status, certification pathway, grade levels taught, years of experience, program size, and if they have children).

Among the respondents, $58.3 \%(n=40)$ were female, $90.3 \%(n=65)$ were white/Caucasian, and they ranged in age from 21 to 65 years old. Additionally, 75\% $(n=54)$ were married and $31.9 \%$ $(n=23)$ had children under the age of 18 . Sixty-one $(84.7 \%)$ were traditionally certified through either a bachelors or master's degree in agricultural education with a student teaching experience and ranged from first year teachers to those with 36 years of experience. When considering grade levels taught, $84.7 \%(n=61)$ taught only high school students (grades 9-12), while the remaining $15.3 \%$ taught either middle school (grades 6-8) or a combination of both middle and high school students. Twenty-eight (38.9\%) respondents reported teaching in a 
single teacher program, with the remaining teaching in multiple teacher programs ranging from two to three teachers.

The data were analyzed using SPSS Version 26 and included descriptive and inferential statistics. For objectives 1, 2, and 3, paired-sample t-tests were implemented to compare SC SBAE teachers self-reported mean scores prior to and during the pandemic to further understand the educational impacts of COVID-19.

\section{Findings}

Findings for Research Question One: Determine the Preparedness of SBAE Teachers to Deliver a Complete SBAE Program Before and During the COVID-19 Pandemic

To determine the preparedness of SBAE teachers in SC, five-items (see Table 1) were scored on a four-point Likert-type scale ( 1 = strongly disagree; 4 = strongly agree) for pre-COVID preparedness and current (during pandemic) preparedness. Table 1 outlines the five items, item means, and standard deviations along with the results of the paired-sample t-tests and calculated effect size.

\section{Table 1}

South Carolina School-based Agricultural Education (SBAE) Teacher Preparedness Prior to and During the COVID-19 Pandemic $(n=72)$

\begin{tabular}{|c|c|c|c|c|c|c|c|}
\hline \multirow{2}{*}{$\begin{array}{l}\text { Item }^{\text {a }} \\
\text { I am prepared to }\end{array}$} & \multicolumn{2}{|c|}{ Pre-COVID } & \multicolumn{2}{|c|}{ Current } & \multirow[b]{2}{*}{$\mathrm{t}$} & \multirow[b]{2}{*}{$p$} & \multirow[b]{2}{*}{$d$} \\
\hline & M & SD & $\mathrm{M}$ & SD & & & \\
\hline $\begin{array}{l}\text { deliver relevant classroom } \\
\text { instruction (in-person) }\end{array}$ & 3.94 & .31 & 3.39 & .77 & 4.88 & $.001^{* *}$ & .69 \\
\hline teach students virtually & 2.24 & 1.1 & 3.04 & .69 & -4.68 & $.001^{* *}$ & .66 \\
\hline $\begin{array}{l}\text { supervise students' } S A E^{b} \\
\text { projects }\end{array}$ & 3.72 & .60 & 2.76 & 1.1 & 5.50 & $.001^{* *}$ & .78 \\
\hline advise FFA members & 3.72 & .61 & 3.00 & .87 & 5.68 & $.001 * *$ & .80 \\
\hline train CDE/LDE teams ${ }^{c}$ & 3.70 & .68 & 2.73 & .94 & 6.61 & $.001 * *$ & .94 \\
\hline
\end{tabular}

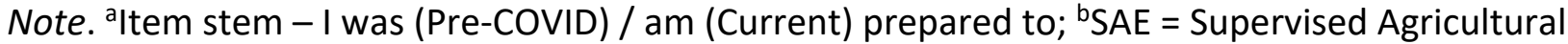
Experience; FFA $=$ National FFA Organization; ${ }^{\mathrm{C} C}$ areer and Leadership Development Events [CDE/LDE] teams. ${ }^{*} p<.05$ or $* * p<.01$.

Each of the five items related to the preparedness of SBAE teachers in SC resulted in a statistically significant $(p<.01)$ difference prior to and during the pandemic, with a decrease in preparedness on all the items expect teaching student virtually, which resulted in a statically significant increase in preparedness. To further understand these differences effect sizes were calculated using Cohen's $d$, resulting in medium effect sizes $(.50<d<.80)$ for preparedness to deliver relevant classroom instruction, teach students virtually, and supervise students' SAE 
projects, while a large effect size $(d>.80)$ was found for advising FFA members and training $C D E / L D E$ teams.

Findings for Research Question Two: Establish Perceived Levels of Self-Efficacy Prior to and During the Pandemic

SBAE teachers in SC varied based on their self-perceived levels of efficacy related to a completed SBAE program (i.e., classroom/laboratory instruction, SAE supervision, FFA advisement) prior to and during the pandemic. Self-efficacy was measured on a sliding scale from 0 (low self-efficacy) to 100 (high self-efficacy) for each of the three components. Scores ranged from a low self-efficacy of 5 for FFA advisement during the pandemic to a high selfefficacy of 100 for classroom and laboratory instruction pre-COVID. Table 2 provides the mean and standard deviation for each of the three components prior to and during the pandemic along with the results of the paired-sample t-tests and calculated effect size.

\section{Table 2}

Self-Efficacy of School-based Agricultural Education (SBAE) Teachers in South Carolina Prior to and During the COVID-19 Pandemic ( $n=72$ )

\begin{tabular}{llllllll}
\hline Item & \multicolumn{2}{l}{ Pre-COVID } & \multicolumn{2}{l}{ Current } & $\mathrm{t}$ & $\mathrm{p}$ & $\mathrm{d}$ \\
\cline { 2 - 8 } & $\mathrm{M}$ & $\mathrm{SD}$ & $\mathrm{M}$ & $\mathrm{SD}$ & & & \\
\hline $\begin{array}{l}\text { Classroom/Laboratory } \\
\quad \text { Instruction }\end{array}$ & 88.43 & 10.54 & 74.21 & 19.58 & 5.70 & $.001^{* *}$ & .82 \\
SAE $^{\text {S Supervision }}$ & 76.19 & 17.86 & 50.02 & 26.66 & 7.20 & $.001^{* *}$ & 1.06 \\
FFA $^{\text {b Advisement }}$ & 88.38 & 11.84 & 61.56 & 24.12 & 7.04 & $.001^{* *}$ & 1.01 \\
\hline
\end{tabular}

Note. ${ }^{\text {a }} \mathrm{SAE}=$ Supervised Agricultural Experience; ${ }^{\mathrm{b} F F A}=$ National FFA Organization. ${ }^{*} p<.05$ or $* * p<.01$.

Participants experienced a decrease in perceived self-efficacy across the three components, as shown by statistically significant decreases $(p<.01)$ and large effect sizes $(d>.80)$.

Additionally, $51.4 \%$ ( $n=37$ ) of respondents felt prepared (probably yes of definitely yes) to deliver online instruction during the pandemic, while the remaining $48.6 \%$ felt that they were probably not or definitely not prepared for the change in instructional delivery. Only $19.4 \%$ ( $n=$ 14) of SBAE teachers in SC that responded had experience prior to the pandemic in delivering online instruction. These factors lead to some of the greatest challenges' respondents faced teaching during the pandemic, including "a lack of hands-on teaching in a virtual environment" which leads to the challenge of making the course "exciting and fun", ultimately impacting student motivation.

Findings for Research Question Three: Determine the Work-Life Balance and Satisfaction of SBAE Teachers Prior to and During the Pandemic

Participants were asked to input their approximate hours worked during an average week on five work related items including, lesson planning, teaching class, grading assignments, SAE 
supervision, and FFA advisement, along with the amount of time dedicated to personal and/or family time. Hours reported ranged from a low of one hour a week spent grading pre-COVID to $11.1 \%(n=8)$ of respondents indicating over 90 hours a week dedicated to family time during the COVID-19 pandemic. Table 3 outlines the mean and standard deviation for hours spent preCOVID and during COVID-19 (current) for each of the six items along with the results of the paired-sample t-tests and effect size.

\section{Table 3}

Average Hours Spent by South Carolina School-based Agricultural Education (SBAE) Teachers Prior to and During the COVID-19 Pandemic ( $n=72)$

\begin{tabular}{llllllll}
\hline Hours Spent & \multicolumn{2}{l}{ Pre-COVID } & \multicolumn{2}{l}{ Current } & $\mathrm{t}$ & $\mathrm{p}$ & $\mathrm{d}$ \\
\cline { 2 - 9 } & $\mathrm{M}$ & $\mathrm{SD}$ & $\mathrm{M}$ & $\mathrm{SD}$ & & & \\
\hline Lesson Planning & 11.00 & 11.79 & 18.18 & 17.52 & -3.86 & $.001^{* *}$ & .61 \\
Teaching & 34.60 & 17.69 & 31.85 & 13.80 & 1.90 & .070 & .30 \\
Grading & 8.98 & 10.56 & 11.57 & 11.85 & -2.71 & $.010^{*}$ & .43 \\
SAE $^{\text {a Supervision }}$ & 10.34 & 12.41 & 8.23 & 13.69 & 1.24 & .220 & .21 \\
FFA $^{\text {b Advisement }}$ & 18.05 & 19.24 & 13.38 & 20.07 & 3.52 & $.010^{*}$ & .56 \\
Personal/Family Time & 30.62 & 25.35 & 32.62 & 27.46 & -1.22 & .230 & .19 \\
\hline
\end{tabular}

Note. ${ }^{\text {a }} \mathrm{SAE}=$ Supervised Agricultural Experience; ${ }^{\mathrm{b} F F A}=$ National FFA Organization. ${ }^{*} p<.05$ or $* * p<.01$.

The sample of responding SBAE teachers reported an increase in hours spent lesson planning ( $p$ $<.01)$, grading $(p<.01)$, and personal/family time $(p>.05)$, while they indicated a decrease in time spent teaching $(p>.05)$, SAE supervision $(p>.05)$, and FFA advisement $(p<.01)$. In addition, a medium effect size $(.50<d<.80)$ was calculated for lesson planning and FFA advisement, while the other four areas resulted in a small effect size $(d<.50)$.

To further understand the work-life balance of SBAE teachers, two questions were asked to address their career satisfaction and life satisfaction pre-COVID and currently (during COVID). Each item was ranked on a five-point Likert-type scale of agreement, where $1=$ extremely dissatisfied and $5=$ extremely satisfied. Sixty-one (84.7\%) participants were either satisfied or extremely satisfied with their career prior to the pandemic, which was then reduced to only $48.6 \%(n=35)$ of SBAE teachers being satisfied or extremely satisfied with their career during the pandemic. Similarly, $81.9 \%(n=59)$ of respondents were satisfied or extremely satisfied with their life prior to COVID-19, while only 68.1\% $(n=49)$ reported to be satisfied or extremely satisfied during the pandemic. Table 4 provides the mean scores and standard deviations for career and life satisfaction prior to and during the COVID-19 pandemic along with the results of the paired-sample t-tests and effect sizes. 
Table 4

Satisfaction of South Carolina School-based Agricultural Education (SBAE) Teachers Prior to and During the COVID-19 Pandemic ( $n=72)$

\begin{tabular}{llllllll}
\hline Satisfaction & \multicolumn{2}{l}{ Pre-COVID } & \multicolumn{2}{l}{ Current } & $\mathrm{t}$ & $\mathrm{p}$ & $\mathrm{d}$ \\
\cline { 2 - 8 } & $\mathrm{M}$ & $\mathrm{SD}$ & $\mathrm{M}$ & $\mathrm{SD}$ & & & \\
\hline Career & 4.15 & 1.01 & 3.10 & 1.25 & 4.53 & $.001^{*}$ & .72 \\
Life & 4.24 & .97 & 3.84 & 1.10 & 2.07 & .050 & .34 \\
\hline
\end{tabular}

${ }^{*} p<.05$ or ${ }^{* *} p<.01$.

Both career and life satisfaction were reported to be lower during the COVID-19 pandemic than before by SBAE teachers in SC. Career satisfaction resulted in a statistically significant difference $(p<.01)$ with a medium $(.50<d<.80)$ effect size. Life satisfaction was also statistically significant $(p=.05)$ but had a small effect size $(d<.50)$, making the difference negligible.

Findings for Research Question Four: Identify the Motivational Factors Driving SBAE Teachers in their Careers

When SC SBAE teachers were asked to rank potential factors that motivate them to teach, $72.2 \%(n=52)$ reported teaching agriculture as the motivating factor. Advising FFA members was the second highest with $25.0 \%(n=18)$ of teachers selecting it as the top factor and another $47.2 \%(n=34)$ identifying it as the second factor. Preparing CDE/LDE teams was only ranked first by one teacher (1.4\%) but was ranked third by $45.8 \%(n=33)$. No teachers indicated working with students SAE projects as being the top motivating factor, instead 41 (56.9\%) identified it as being the least motivating of the four potential factors.

\section{Conclusions, Discussion, and Recommendations}

SBAE teachers in SC feel less prepared to deliver relevant classroom instruction, supervise students' SAE projects, advise FFA members, and train CDE/LDE teams know then they did prior to the COVID-19 pandemic, while they feel more prepared to teach student virtually than ever before. This aligns with previous research identifying teachers and their school districts as not being prepared for the shift to online or hybrid instructional models (Daniel, 2020). Although the more teachers interacted with learning management systems and virtual teaching platforms (i.e., Zoom or Google Meets) the more prepared they felt, unfortunately, in most cases this was taking away from their time and preparedness in other areas (Bushweller, 2020). When evaluating SBAE teachers' self-efficacy of a complete SBAE program (i.e., classroom/laboratory instruction, FFA, and SAE), statistically significant decreases $(p<.01)$ and large effect sizes $(d>.80)$ were found across all three components. The pandemic forced a shift in many of the traditional roles of an SBAE teacher, as identified by Terry and Briers (2010), as these roles morphed, teachers felt less efficacy than before, which brought about many challenges for the teachers. Thus, teachers began to question what they were doing and how effective they were in their chosen career. The human capital including the education, skills, 
experiences, and trainings (Becker, 1964; Little, 2003; Schultz, 1971; Smith, 2010; Smylie, 1996) they have received over the years that was essential for their daily job functions, was perhaps, no longer adequate.

These sudden changes in the job duties impacted the preparedness and self-efficacy of SBAE teachers, impacting both their career and personal life satisfaction. Career satisfaction experienced the greatest impact and resulted in a statistically significant difference $(p<.01)$ with a medium effect size. This aligns with the recent work of McKim and Sorenson (2020) who found a significant decrease in SBAE teaching satisfaction because of the COVID-19 pandemic. Conceivably this relates to the increase in time needed to lesson plan and grade student work during the pandemic compared to pre-pandemic and the reduction in time spent teaching, which was found to be the primary reason participants selected a career as an SBAE teacher. Life satisfaction on the other hand resulted in a negligible difference ( $p=.05$, small effect size), which perhaps can be related to the reduced time spent teaching, conducting SAE visits, and advising the FFA chapter along with the additional time reported for personal/family time. For many, teaching SBAE is life, linking the impact on both work and life satisfaction found in this study.

The COVID-19 pandemic has impacted SBAE teachers in SC in a variety of ways, from their instructional delivery methods and FFA and SAE responsibilities to time spent teaching versus time spent with family. SBAE teachers look forward to returning to a sense of normalcy with inperson teaching and FFA events, but this is also a time to reflect on potential positive outcomes from the pandemic (i.e., reduced weekly workload and increased personal/family time). Moving forward SBAE teachers should evaluate their roles and responsibilities associated with their career and determine how to best bring balance into their lives, as the environmental factors (see Figure 1), including the COVID-19 pandemic caused a disruption in the status quo of the necessary human capital required for their career. Therefore, SBAE teacher educators, agricultural education state staff, and school administrators should consider the demands placed on SBAE teachers and determine how to best support them, preparing teachers for longevity in a successful career. Additionally, functions that became essential during the pandemic (i.e., virtual program delivery, effective online instruction, hosting virtual meetings, and operating learning management systems), should become an integral part of teacher preparation programs in the future to better prepare future teachers for an ever-changing educational climate.

Additional research on the roles and responsibilities of SBAE teachers post-pandemic should be conducted to better understand the impact of COVID-19 on the profession. Online instruction was immediately implemented for teachers across the country, therefore, further investigation into effective online delivery for SBAE programs is essential. Finally, this study should be adapted to evaluate teachers post-pandemic through a then-now design, allowing participants to reflect on their experiences during the COVID-19 pandemic (then), while also reporting their current experiences teaching SBAE post-pandemic (now) on the same items. 


\section{References}

Banerjee, N., Stearns, E., Moller, S., \& Mickelson, R. A. (2017). Teacher job satisfaction and student achievement: The roles of teacher professional community and teacher collaboration in schools. American Journal of Education, 123(2), 203-241. https://doi.org/10.1086/689932

Becker, G. S. (1964). Human capital: A theoretical and empirical analysis with special reference to education. National Bureau of Economic Research.

Bushweller, K. (2020). How COVID-19 is shaping tech use. What that means when schools reopen. Education Week. https://www.edweek.org/ew/articles/2020/06/03/howcovid19-is-shaping-tech-usewhat.html

Centers for Disease Control and Prevention [CDC]. (2020). Situation summary. https://www.cdc.gov/coronavirus/2019-ncov/cases-updates/summary.html

Daniel, S. J. (2020). Education and the COVID-19 pandemic. Prospects, 49, 91-96. https://doi.org/10.1007/s11125-020-09464-3

Dillman, D. A., Smyth, J. D., \& Christian, L. M. (2014). Internet, phone, mail, and mixed-mode surveys: The tailored design method (4th ed.). Wiley.

John, O. P., \& Robins, R. W. (1994). Accuracy and bias in self-perception: Individual differences in self-enhancement and the role of narcissism. Journal of Personality and Social Psychology, 66(1), 206-219. https://doi.org/10.1037/0022-3514.66.1.206

Lepak, D. P., \& Snell, S. A. (1999). The human resource architecture: Toward a theory of human capital allocation and development. Academy of Management Review, 24(1), 31-48. https://doi.org/10.5465/amr.1999.1580439

Lindner, J., Clemons, C., Thoron, A., \& Lindner, N. (2020). Remote instruction and distance education. A response to COVID-19. Advancements in Agricultural Development, 1(2), 53-64. https://doi.org/10.37433/aad.v1i2.39

Lindner, J. R., Murphy, T. H., \& Briers, G. E. (2001). Handling nonresponse in social science research. Journal of Agricultural Education, 42(4), 43-53. https://doi.org/10.5032/ihae.2001.04043

Little, J. W. (2003). Inside teacher community: Representations of classroom practice. Teachers College Record, 105(6), 913-945. https://www.tcrecord.org/content.asp?contentid=11544 
McKim, A. J., \& Sorenson, T. J. (2020). Agricultural educators and the pandemic: An evaluation of work and life variables. Journal of Agricultural Education, 61(4), 214-228. https://doi.org/10.5032/jae.2020.04214

National FFA Organization. (2015). Agricultural education. https://www.ffa.org/agriculturaleducation/

Privitera, G. J. (2020). Research methods for the behavioral sciences (3rd ed.). Sage.

Schultz, T. W. (1971). Investment in human capital: The role of education and of research. The Free Press.

Smith, E. (2010). Sector-specific human capital and the distribution of earnings. Journal of Human Capital, 4(1), 35-61. https://doi.org/10.1086/655467

Smylie, M. A. (1996). From bureaucratic control to building human capital: The importance of teacher learning in education reform. Educational Researcher, 25(9), 9-11. https://doi.org/10.3102/0013189X025009009

Terry, R., Jr., \& Briers, G. E. (2010). Roles of the secondary agriculture teacher. In R. Torres, T. Kitchel, \& A. Ball (Eds.), Preparing and advancing teachers in agricultural education (pp. 86-98). Columbus: Curriculum Materials Service, The Ohio State University.

Torres, R. M., Ulmer, J. D., \& Aschenbrener, M. S. (2008). Workload distribution among agriculture teachers. Journal of Agricultural Education, 49(2), 75-87. https://doi.org/10.5032/jae.2008.02075

Wilson, S. M., \& Ball, D. L. (1996). Helping teachers meet the standards: New challenges for teacher educators. The Elementary School Journal, 97(2), 121-138. https://doi.org/10.1086/461858

World Health Organization. (2020). Coronavirus disease 2019 (COVID-19) situation report51. https://www.who.int/docs/default-source/coronaviruse/situationreports/20200311-sitrep-51-covid-19.pdf'sfvrsn=1ba62e57 10

(C) 2021 by authors. This article is an open access article distributed under the terms and conditions of the Creative Commons Attribution license (http://creativecommons.org/licenses/by/4.0/). 